\title{
Functional Prosthetic Spacer Surgical Technique for Definitive Management of Hip Periprosthetic Joint Infection
}

\author{
Jordan $R$ Bonier, DO, MS $S^{1}$, Ahmed Siddiqi, DO, $M B A^{2^{*}}$, Ronald E Delanois, $M D^{1}$ and James Nace, DO, \\ FAOAO, MPT \\ ${ }^{1}$ Rubin Institute for Advanced Orthopedics, Sinai Hospital of Baltimore, USA \\ ${ }^{2}$ Department of Orthopedics, Cleveland Clinic Foundation, Cleveland, USA

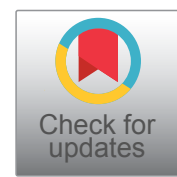

\begin{abstract}
Introduction: Periprosthetic joint infection (PJI) of the hip has been treated with two-stage revision arthroplasty with success. Concerns with this treatment approach include significant morbidity, cost, and functional impairment for the patient. One-stage revision arthroplasty for PJI is commonly performed outside of the United States.

Materials and methods: We review a novel surgical technique for functional articulating antibiotic cement spacers of the hip with the goal of eradicating infection, reducing morbidity of multiple surgical procedures, improving patient function, and decreasing cost.

Results: The appropriately sized acetabular polyethylene liner is utilized. If instability is a concern, a dual mobility liner can be utilized. The back side of either chosen liner is scored with a saw or burr. In situations where significant acetabular bone loss is present, screws may be placed in the anterior and/or posterior columns to serve as rebar for the planned cement mantle. The femur is broached sequentially until an adequate fit is obtained. A cemented femoral stem is used primarily. If proximal femoral bone stock is poor, a long-cemented stem is utilized.
\end{abstract}

Discussion: One-stage revision arthroplasty with a functional antibiotic cement spacer has been used to treat of PJI of the knee with success, while avoiding complications and cost associated with prefabricated and manufactured spacers.

\section{Keywords}

Functional prosthetic spacers, Functional one-stage, Two-stage, PJI, THA PJI

\section{Introduction}

Periprosthetic joint infection (PJ) is a devastating complication causing significant morbidity, mortality, and healthcare expenditure $[1,2]$. With the steady increase in total hip arthroplasty (THA) performed annually in the United States, effective hip PJI management is of paramount importance $[3,4]$. Currently, the gold standard for definitive chronic PJI is two-stage revision arthroplasty [5]. However, this treatment is associated with significant morbidity, cost, and functional impairment for the patient [5-9]. Alternatively, one-stage revision arthroplasty has shown promising results in the literature and is widely practiced outside of the United States [10-12]. Although appealing, prefabricated manufactured antibiotic molds and spacers are attributed with limited patient function, greater theoretical risk of mechanical failure and significant cost [13-17].

The use of functional prosthetic spacers as a modified onestage exchange arthroplasty has recently gained momentum with success for the management of knee PJI [6,18-21]. This technique has the advantages of reducing the morbidity of multiple surgical procedures within a short time frame, immediate improvement of patient function, non-inferior infection eradication compared with two-stage exchange and notable cost-saving from potential prevention of a secondary procedure $[18,21]$. Therefore, the purpose of this article is to re-

*Corresponding author: Ahmed Siddiqi, DO, MBA, Department of Orthopedics, Cleveland Clinic Foundation, 9500 Euclid Avenue/ND20, Cleveland, OH, 44195, USA

Accepted: March 23, 2021

Published online: March 25, 2021

Citation: Bonier JR, Siddiqi A, Delanois RE, et al. (2021) Functional Prosthetic Spacer Surgical Technique for Definitive Management of Hip Periprosthetic Joint Infection. J Orthop Surg Tech 4(1):254-261

Copyright: (c) 2021 Bonier JR, et al. This is an open-access article distributed under the terms of the Creative Commons Attribution License, which permits unrestricted use, distribution, and reproduction in any medium, provided the original author and source are credited. 


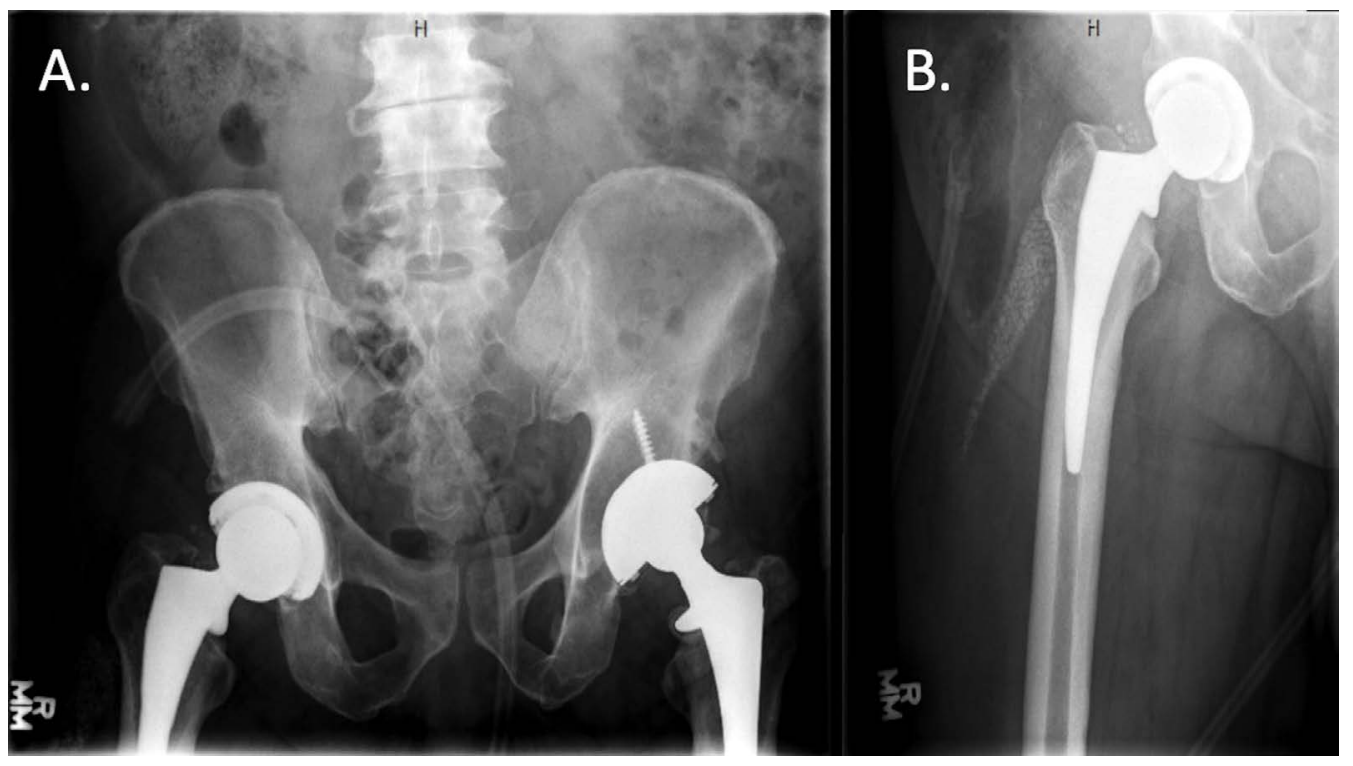

Figure 1: Pre-operative X-rays. A. Anteroposterior (AP) pelvis radiograph (a) and AP hipviews demonstrating a right total hip replacement. The patient was diagnosed with a periprosthetic right hip infection. Explant and placement of a functional prosthetic spacer was planned.

view our surgical technique, present procedures for complex cases, and offer coding guidelines for functional prosthetic spacers for the definitive management of chronic hip PJI.

\section{Materials and Methods}

Institutional Review Board approval was except for this surgical technique review. Patients are placed in the lateral decubitus position securely using standard positioners with padding ofall bony prominence. Leg lengths and hip flexor and abductor tension is assessed prior to the procedure for intraoperative trialing comparison (Figure 1). The operative extremity is prepped and draped using standard protocols. Surgical exposure is performed through any standard hip approach. The senior author's preferred surgical approach is a modified Watson-Jones anterolateral approach due to its inherent stability and ability to repair or reconstruct any concurrent abductor dysfunction.

After hip dislocation and femoral head removal, an extensive excisional debridement is carried out; ensuring all devitalized tissues is adequately removed. Frozen sections are not routinely obtained and reserved in situations with questionable PJI criteria based on the 2014 Musculoskeletal Infection Society (MSIS) definition [22]. A minimum of five deep tissue cultures from the acetabulum and femur are obtained.

\section{Results}

\section{Surgical technique}

Attention is typically focused on extraction of the femoral component to aid in acetabular exposure. A combination of flexible osteotomes, radial osteotomes, double-sided reciprocating saw, and Kirschner wires are used to facilitate removal of the femoral stem. The goal is to safely extract the stem and preserve proximal bone stock which may be compromised in chronic infectious processes. A burr can be utilized along the proximal femur to gain access to the top of the stem, partic- ularly if there is an over-hanging greater trochanter or varus subsidence of the stem. If the stem is well in-grown distally, an extended trochanteric osteotomy is utilized to gain access to the distal aspect of the stem. The presence of a fully coated cylindrical stem may require utilization of a metal-cutting burr and trephine reamers to remove the stem. The cement mantle of a cemented stem is disrupted and removed with a cement removal set, including osteotomes, specialized hooks, taps and long reverse curettes. If necessary, sharp reamer/ drill and, rarely, an ultrasonic cement extractor device can be utilized in special circumstances when the former techniques are unsuccessful. Special attention should be taken when reaming or drilling to not allow the cement mantle to cause the drill to deviate from the intramedullary canal. Fluoroscopic imaging can be employed to confirm reaming trajectory and to remove all polymethyl methacrylate material from the femoral canal.

Attention is then placed on the acetabular component. After adequate exposure is obtained, the liner is first removed with an osteotome and mallet along one edge of the liner and driven into the opposite edge of the poly liner. Acetabular screws are removed if present. The stability of the acetabular shell is assessed with the implant-specific inserter tool attached. A few gentle in-line taps with the mallet and extractor tool can assess stability. If the acetabular component is well-fixed, the shell is removed with sequential oscillating curved blades using either motorized or manual acetabular extraction systems. A Cobb elevator, curette, and acetabular reamers are used to debride any devitalized tissue from the bony surface of the acetabulum.

Full strength povidone-iodine solution is applied to the wound and all bony surfaces. An acetabular reamer on power is used to debride the acetabulum, taking care to preserve the anterior and posterior columns and medial wall. A femoral canal brush with the handle cut and chucked onto a power drill is used to debride the femoral canal (Figure 2). The 


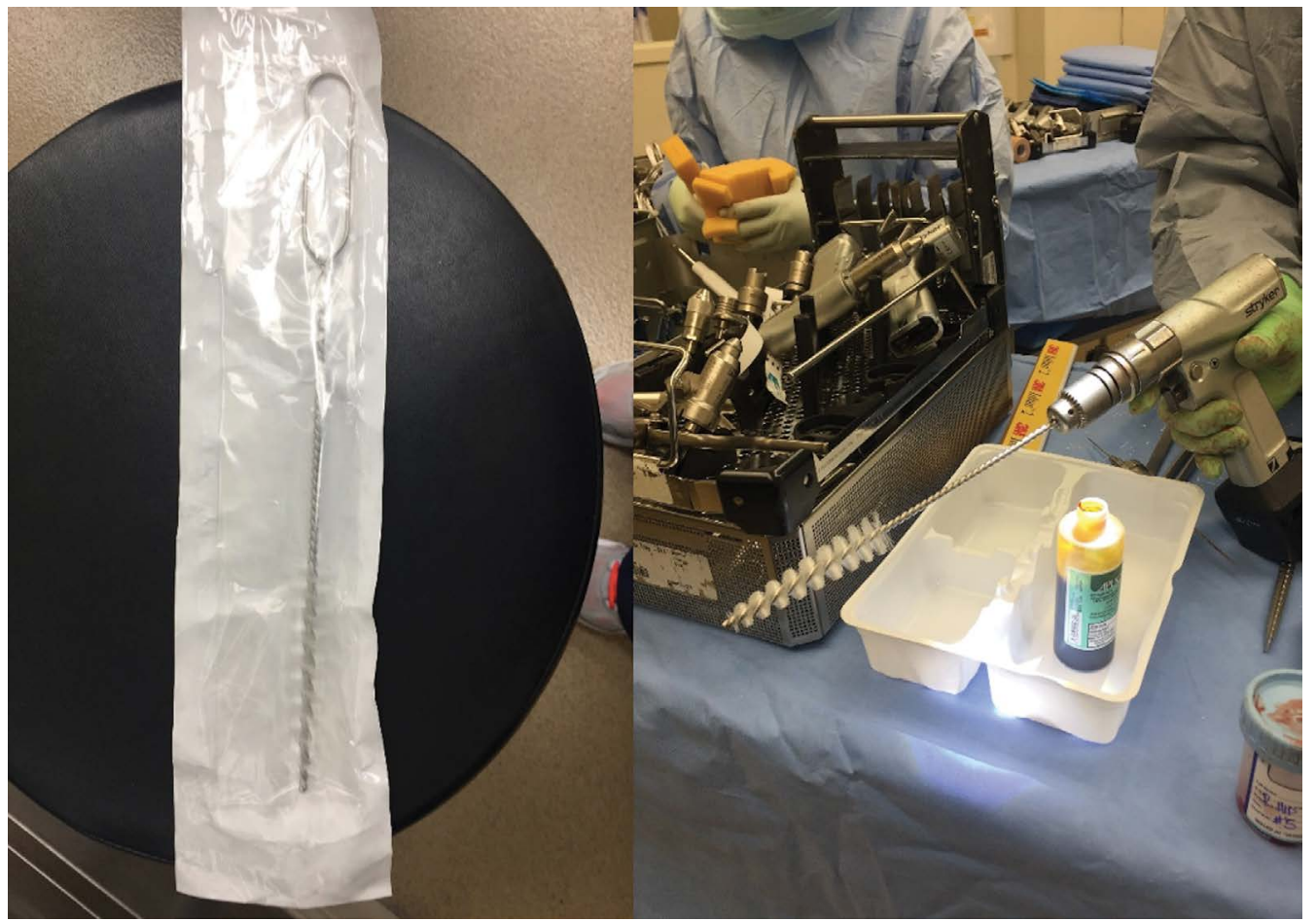

Figure 2: Femoral canal brush. The handle is cut and chucked onto a power drill and used to debride the femoral canal.

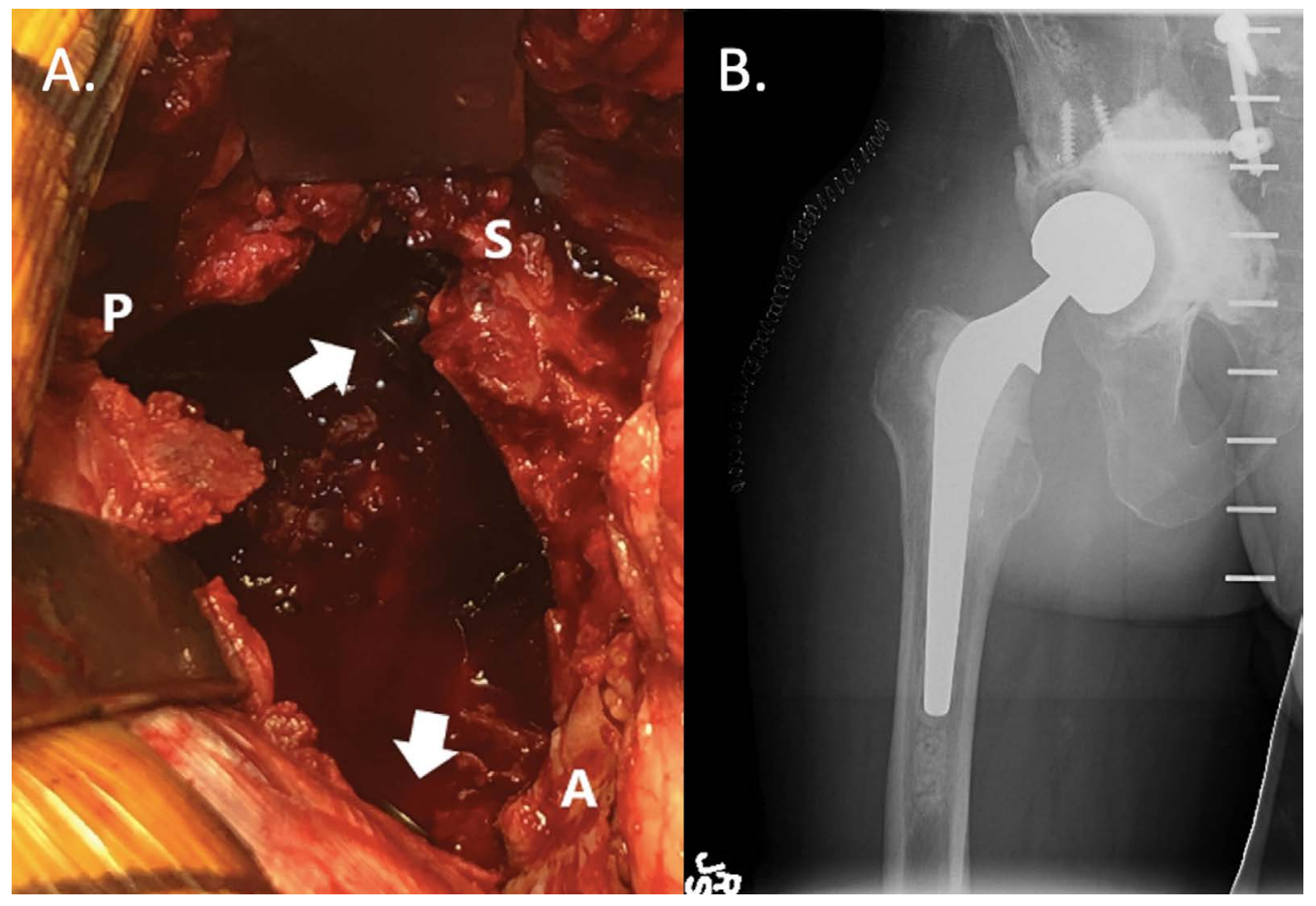

Figure 3: A) Severe acetabular bone loss with complete absence of the medial wall from infectious bony compromise. $6.5 \mathrm{~mm}$ cancellous acetabular Screws were placed in the pubis and ilium (arrows) to serve as rebar for cement mantle. A: Anterior, P: Posterior, S: Superior; B) Postoperative anteroposterior radiograph demonstrating functional prosthetic hip spacer with two rebar screws.

wound and bony surfaces are irrigated with normal saline.

Trial acetabular liners are sequentially trialed in the acetabulum until an intimate fit is obtained. The final polyethy lene liner selected for the acetabular component is typically one size smaller than the last trial liner to ensure an adequate
( $\sim 3 \mathrm{~mm}$ ) cement mantle for the final acetabular component. The one size larger trial liner is left in place to facilitate stability while trialing.

The femur is broached sequentially until an adequate fit is obtained. A cemented femoral stem trial is placed. If prox- 
imal femoral bone stock is poor, a long-cemented stem trial is utilized. Distal reaming may be necessary in this instance. Femoral head trial is placed, and the hip is carefully reduced into the trial liner. When appropriate leg length and offset are achieved, the height of the femoral stem is measured from a fixed anatomical point, usually the greater trochanter as a reference for the appropriate height when cementing. The leg length is restored by assessing the previous stem height, preoperative templating, and assessing muscle/tendon tension compared to preoperative assessment. The hip is dislocated, and trial components removed.
The wound and all bony surfaces are copiously irrigated with minimal 9 liters of normal saline. The entire surgical team re-scrubs, re-gowns, re-gloves, and obtains new sterile instruments, and the patient is re-prepped and re-draped as if starting a new surgical case. The appropriately sized acetabular polyethylene liner is utilized. If instability is a concern, a dual mobility liner can be utilized. The backside of either chosen liner is scored with a saw or burr. In situations where significant acetabular bone loss is present, screws may be placed in the anterior and/or posterior columns to serve as rebar for the planned cement mantle (Figure 3). On the back table, an

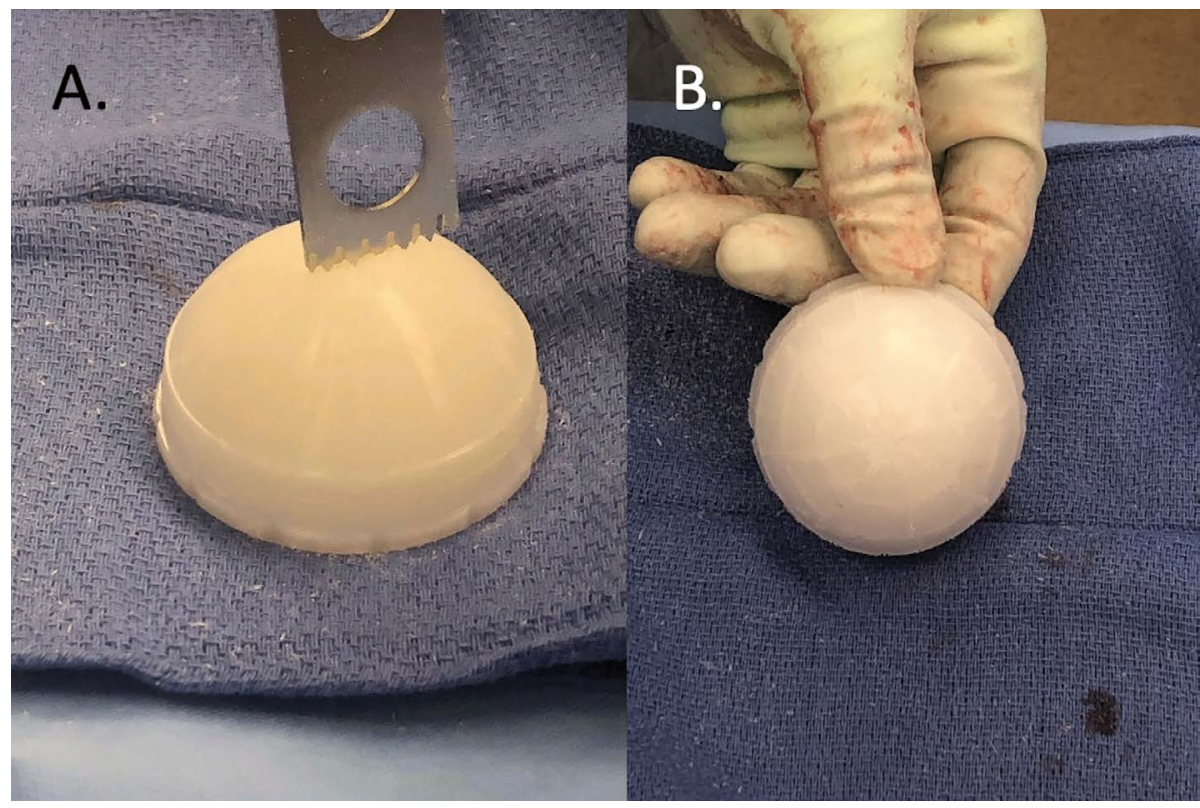

Figure 4: Polyethylene liner preparation. A) The backside of a polyethylene liner is scored on the back table with an oscillating saw; B) Prepared polyethylene liner prior to cementation.

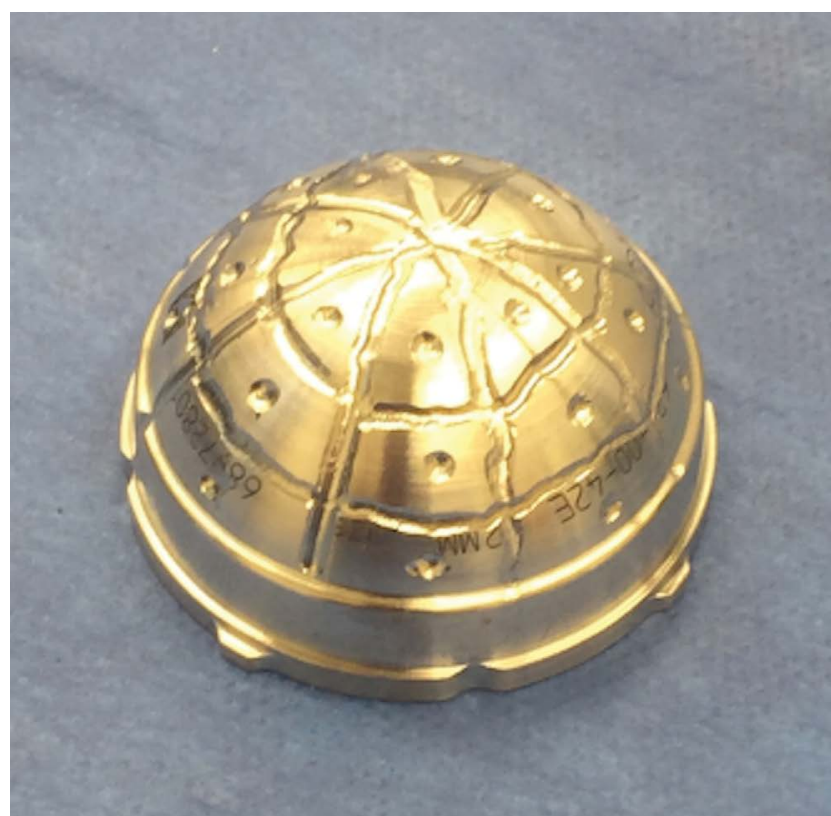

Figure 5: Dual mobility liner preparation. A cobalt-chrome liner for a dual mobility bearing is scored on the back table with a highspeed burr. The authors prefer to use this option in patients at high risk for instability. 
Citation: Bonier JR, Siddiqi A, Delanois RE, et al. (2021) Functional Prosthetic Spacer Surgical Technique for Definitive Management of Hip Periprosthetic Joint Infection. J Orthop Surg Tech 4(1):254-261

oscillating saw or burr is used to score the backside of the liner to improve cement interdigitation (Figure 4 and Figure 5).

Antibiotic calcium sulfate beads are routinely placed in the femoral canal before cementation and throughout the intracapsular space after final reduction. The final femoral stem is cemented by hand into the femoral canal to ensure adequate space for cement mantle, as well as un obstructed passage.

Low viscosity polymethyl methacrylate cement isprepared by hand-mixing and augmented with culture specific, heat stabile antibiotics. The authors prefer an approximate $10 \%$ concentration of total antibiotic concentration. Typically, 2 grams of vancomyc in and 1.2 grams of to bramyc in are added per 40 -gram bag of cement. All attempts are made to customize the antibiotics for organism-specific antibiotic [23] or antifungal agents [24]. A total of 3 bags of cement are divided equally for the acetabular and femoral components. The cement is first mixed with solvent and powder, then antibiotic slowly added to the mix to facilitate drug elution [25]. Our $10 \%$ antibiotic cement concentration is greater than previously described antibiotic cement concentrations used in one-stage revision arthroplasties [11]. A high antibiotic cement concentration has theoretical concern from increased rate of mechanical failure from cement fragility [26,27], although some studies report no difference in mechanical strength $[28,29]$. Further investigation and longer-term follow-up is needed to evaluate the longevity of high-dose antibiotic cement.

The acetabular liner is placed with the cement in the doughy phase (Figure 6) after cleansing and drying the acetabulum. Appropriate abduction angle is achieved using the preoperative template and the amount of superior under-coverage of the liner as a guide. Appropriate anteversion is achieved using the transverse acetabular ligament and the anterior and posterior walls as a reference. Adequate fixation

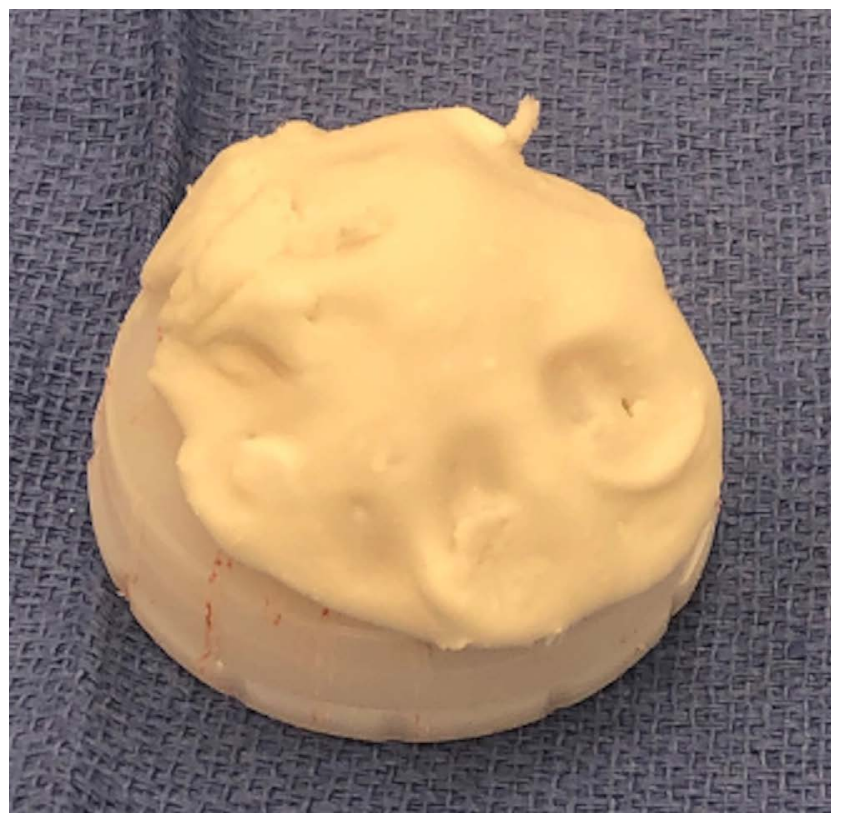

Figure 6: Cement preparation. Antibiotic-laden cement is placed on the backside of a polyethylene liner and on the acetabulum prior to insertion. requires steady and light manual pressure to be held until the cement completely cures, while removing any excess cement. Special care must be taken not place the acetabular liner superiorly or too medially, to prevent raising the hip center, decreasing offset, or creating an uneven cement mantle. Once cured, excess cement around the rim is carefully removed with an osteotome, making sure not to disrupt the cement mantle and compromise fixation. Please note that acetabular retractor placement is critical to ensure that no cement contacts the retractors, as this can cause premature liner pullout when removing the retractors.

Cement for femoral fixation is then prepared and mixed by hand as noted above after cleansing and drying the femoral intramedullary canal. Cement is placed around the femoral component in the doughy phase and packed proximally. A lap sponge is placed in the acetabulum. The femoral component is inserted to the appropriate height based on measurement during trialing. The appropriate femoral anteversion is achieved, excess cement is removed, and the component is held in place until cement cures (Figure 7). Femoral heads are carefully trialed, and the appropriately sized femoral head is impacted, and the hip is reduced. The authors preferred technique is to trial one head ball size below the final component to avoid any excessive pressure across the freshly cemented liner. For instance, if a 44-millimeter liner is utilized, we will trial with a 40-millimeter femoral head ball to assess length and tension of the hip. The wound is irrigated. Remaining an-

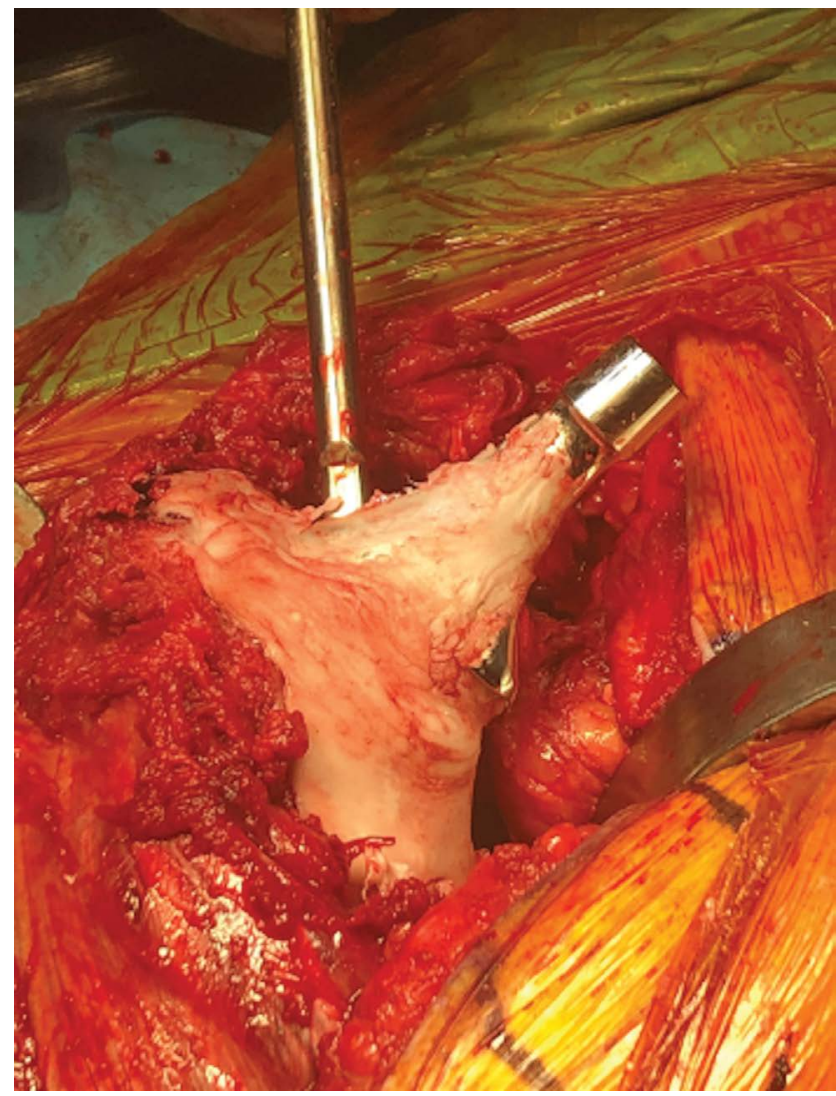

Figure 7: Femoral component insertion. The femoral component is inserted to the appropriate height. The appropriate femoral anteversion is achieved and held in place until cement cures. 


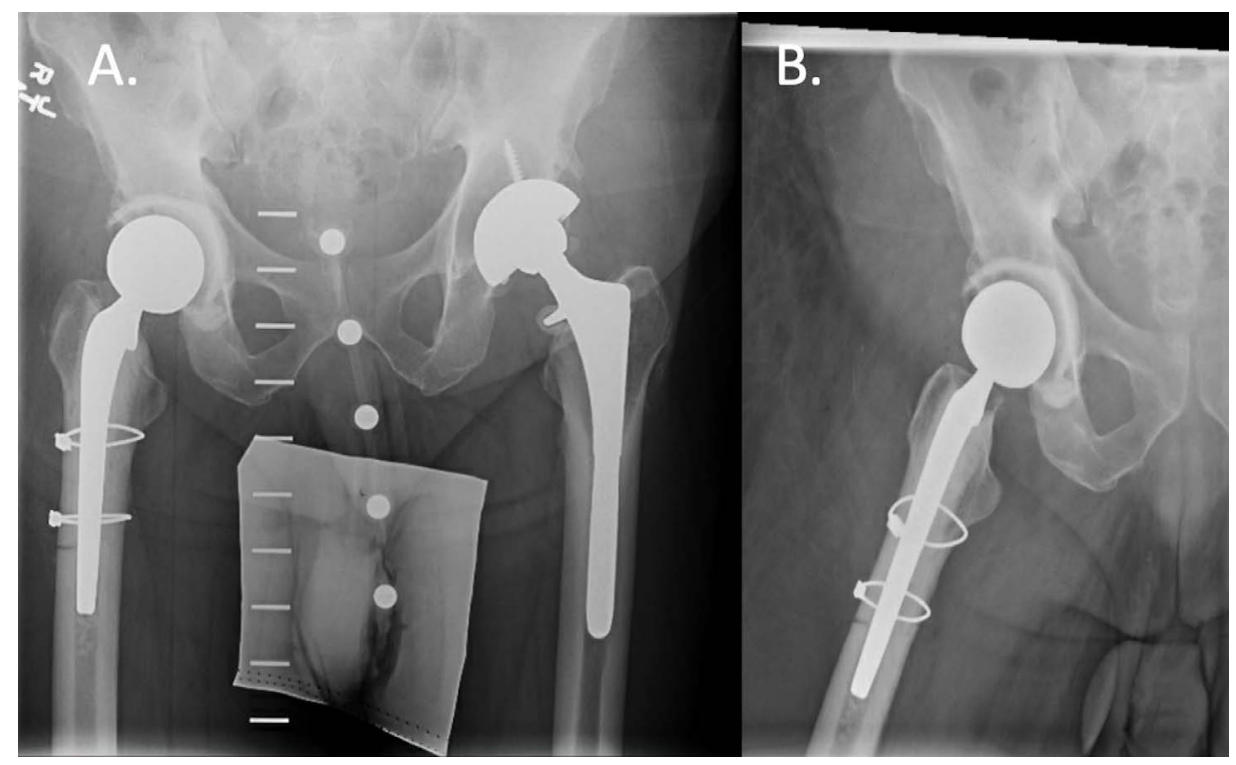

Figure 8: Post-operative x-rays. A) AP pelvis and B) Frog leg lateral right hip views demonstrating a right hip functional prosthetic spacer. The well-fixed in situ femoral stem required extended trochanteric osteotomy for removal.

Table 1: Delphia criteria for success after treatment of periprosthetic joint infection [33].

1. Infection eradication characterized by a healed wound without fistula, drainage, or pain, and no infection recurrence caused by the same organism strain.

2. No subsequent surgical intervention for infection after reimplantation surgery.

3. No occurrence of PJI-related mortality (i.e., due to sepsis and necrotizing fasciitis).

Table 2: New 2020 CPT codes for drug delivery device implantation.

\begin{tabular}{|l|l|l|}
\hline CPT Code & CPT Code Inclusion \\
\hline 20700 & $\begin{array}{l}\text { Manual preparation and insertion of drug-delivery device(s); deep; usually includes polymethylmethacrylate } \\
\text { beads with calcium sulfate, tobramycin or vancomycin }\end{array}$ \\
\hline 20702 & $\begin{array}{l}\text { - } \\
\text { - }\end{array}$ & List separately in addition to primary procedure \\
\hline 20704 & $\begin{array}{l}\text { - Manual preparation and insertion of drug-delivery device(s), intramedullary } \\
\end{array}$ & - Manual preparation and insertion of drug-delivery device(s), intra-articular \\
\hline
\end{tabular}

CPT: Current Procedural Terminology

tibiotic beads are placed intra-capsular. Standard closure is performed (Figure 8).

\section{Discussion}

For our post-operative protocol, weight bearing as tolerated is typically allowed. Broad-spectrum antibiotics are continued intravenously and transitioned to culture-specific therapy for 6 weeks, on average [30]. Serial serum ESR and CRP levels are obtained until levels normalize or considerably downtrend towards normalization [31], at which point IV antibiotics are discontinued anda subsequent three month course of oral antibiotics is considered especially for higher-risk patients [32]. Second-stage revision is not considered unlesstreatment failure occurs as defined by the Delphi criteria (Table 1) [33]. Well-functioning articulating spacers are retained with periodic clinical and radiographic follow up.
For coding purposes, the operative procedure should be described as "total hip arthroplasty revision with permanent prosthesis utilizing hand-crafted antibiotic cement with extensive excisional debridement including deep tendon, tissue, and bone." This description allows for appropriate coding of a revision arthroplasty versus antibiotic spacer, which has a significantly different coding and billing reimbursement. Recent changes in coding including the "hand-crafted cement" were made in 2020 (Table 2) [34].

Of note, some first stage prefabricated implants have recently under gone revalidation and may not even be available for use [35]. In addition, these implants can be costly [36]. This has led to some challenges for both the surgeon and hospital. The above implants are readily available, inexpensive, and avoid concerns associated with mechanical failure of prefabricated and manufactured antibiotic spacers [13-17]. 
Citation: Bonier JR, Siddiqi A, Delanois RE, et al. (2021) Functional Prosthetic Spacer Surgical Technique for Definitive Management of Hip Periprosthetic Joint Infection. J Orthop Surg Tech 4(1):254-261

\section{Summary}

As healthcare reform has shifted to placing greater focus on defending procedural value based on quality of care relative to cost, there is greater emphasis on improving patient outcomes [37]. Since chronic hip PJI remains a significant burden on the healthcare system, management with onestage revision arthroplasty has gained momentum by offering non-inferior clinical outcomes to conventional two-stage exchange arthroplasty and reducing cost $[18,21]$. This is the first paper, to our knowledge, that presents a detailed surgical technique for functional articulating antibiotic cement spacers by reducingpatient morbidity, improving patient function, and decreasing cost, while providing an effective treatment for prosthetic joint infection of the hip.

\section{Acknowledgment and Funding Sources}

None.

\section{References}

1. Joseph TN, Chen AL, Di Cesare PE (2003) Use of antibiotic-impregnated cement in total joint arthroplasty. J Am Acad Orthop Surg 11: 38-47.

2. Peersman G, Laskin R, Davis J, et al. (2001) The Insall award paper: Infection in total knee replacement: A retrospective review of 6489 total knee replacements. Clinical Orthopaedics and Related Research 392: 15-23.

3. Sloan M, Premkumar A, Sheth NP (2018) Projected volume of primary total joint arthroplasty in the u.s., 2014 to 2030. J Bone Jt Surg Am 100: 1455-1460.

4. Kurtz S, Ong K, Lau E, et al. (2007) Projections of primary and revision hip and knee arthroplasty in the United States from 2005 to 2030. J Bone Jt Surg Ser Am 89: 780-785.

5. Cui Q, Mihalko WM, Shields JS, et al. (2007) Antibiotic-impregnated cement spacers for the treatment of infection associated with total hip or knee arthroplasty. J Bone Jt Surg Ser Am 89: 871-882.

6. Marson BA, Walters ST, Bloch B, et al. (2018) Two stage revision surgery for infected total knee replacements: Reasonable function and high success rate with the use of primary knee replacement implants as temporary spacers. Eur J Orthop Surg Traumatol 28: 109-115.

7. Kallala RF, Ibrahim MS, Sarmah S, et al. (2015) Financial analysis of revision knee surgery based on NHS tariffs and hospital costs does it pay to provide a revision service? Bone Jt J 97: 197-201.

8. Kim TWB, Makani A, Choudhury R, et al. (2012) Patient-reported activity levels after successful treatment of infected total knee arthroplasty. J Arthroplasty 27: 81-85.

9. Gomez MM, Tan TL, Manrique J, et al. (2015) The fate of spacers in the treatment of periprosthetic joint infection. J Bone Jt Surg Am 97: 1495-1502.

10. Vaishya R, Agarwal AK, Rawat SK, et al. (2017) Is single-stage revision safe following infected total knee arthroplasty? Cureus 9: e1629.

11. Haddad FS, Sukeik M, Alazzawi S (2015) Is single-stage revision according to a strict protocol effective in treatment of chronic knee arthroplasty infections? Clin Orthop Relat Res 473: 8-14.

12. Tibrewal S, Malagelada F, Jeyaseelan L, et al. (2014) Single-stage revision for the infected total knee replacement: Results from a single centre. Bone Jt J 96: 759-764.

13. Corona PS, Barro V, Mendez M, (2014) Industrially prefabricated cement spacers: Do vancomycin-and gentamicin-impregnated spacers offer any advantage? Clin Orthop Relat Res 472: 923932.

14. Sabry FY, Szubski CR, Stefancin JJ, et al. (2013) Comparison of complications associated with commercially available and custom-made articulating spacers in two-stage total hip arthroplasty revision. Curr Orthop Pract 24: 406-413.

15. Anagnostakos K, Jung J, Schmid NV, et al. (2009) Mechanical complications and reconstruction strategies at the site of hip spacer implantation. Int J Med Sci 6: 274-279.

16. Faschingbauer $M$, Reichel $H$, Bieger $R$, et al. (2015) Mechanical complications with one hundred and thirty eight (antibiotic-laden) cement spacers in the treatment of periprosthetic infection after total hip arthroplasty. Int Orthop 39: 989-994.

17. Erivan R, Lecointe T, Villatte G, et al. (2018) Complications with cement spacers in 2-stage treatment of periprosthetic joint infection on total hip replacement. Orthop Traumatol Surg Res 104: 333-339.

18. Siddiqi A, George NE, White PB, et al. (2018) Articulating spacers as a modified one-stage revision total knee arthroplasty: A preliminary analysis. Surg Technol Int 32: 239-248.

19. Petis SM, Perry KI, Pagnano MW, et al. (2017) Retained antibiotic spacers after total hip and knee arthroplasty resections: high complication rates. J Arthroplasty 32: 3510-3518.

20. Choi HR, Freiberg AA, Malchau H, et al. (2014) The fate of unplanned retention of prosthetic articulating spacers for infected total hip and total knee arthroplasty. J Arthroplasty 29: 690-693.

21. Siddiqi A, Nace J, George NE, et al. (2019) Primary total knee arthroplasty implants as functional prosthetic spacers for definitive management of periprosthetic joint infection: A Multicenter Study. J Arthroplasty 34: 3040-3047.

22. Parvizi J, Gehrke T (2014) Definition of periprosthetic joint infection. J Arthroplasty 29: 1331.

23. Anagnostakos K (2016) Therapeutic use of antibiotic-loaded bone cement in the treatment of hip and knee joint infections. J Bone Jt Infect 2: 29-37.

24. Nace J, Siddiqi A, Talmo CT, et al. (2019) Diagnosis and management of fungal periprosthetic joint infections. J Am Acad Orthop Surg 27: e804-e818.

25. Shahpari O, Mousavian A, Elahpour N, et al. (2020) The use of antibiotic impregnated cement spacers in the treatment of infected total joint replacement: Challenges and achievements. Arch Bone Jt Surg 8: 11-20.

26. Dunne NJ, Hill J, McAfee P, et al. (2008) Incorporation of large amounts of gentamicin sulphate into acrylic bone cement: Effect on handling and mechanical properties, antibiotic release, and biofilm formation. Proc Inst Mech Eng Part H J Eng Med 222: 355-365.

27. Pelletier MH, Malisano L, Smitham PJ, et al. (2009) The compressive properties of bone cements containing large doses of antibiotics. J Arthroplasty 24: 454-460.

28. Klekamp J, Dawson JM, Haas DW, et al. (1999) The use of vancomycin and tobramycin in acrylic bone cement: Biomechanical effects and elution kinetics for use in joint arthroplasty. J Arthroplasty 14: 339-346. 
29. He Y, Trotignon JP, Loty B, et al. (2002) Effect of antibiotics on the properties of poly (methylmethacrylate)-based bone cement. J Biomed Mater Res 63: 800-806.

30. Sousa R, Abreu MA (2018) Treatment of prosthetic joint infection with debridement, antibiotics and irrigation with implant retention - a narrative review. J Bone Jt Infect 3: 108-117.

31. Lindsay CP, Olcott CW, Del Gaizo DJ (2017) ESR and CRP are useful between stages of 2-stage revision for periprosthetic joint infection. Arthroplast Today 3: 183-186.

32. Frank JM, Kayupov E, Moric M, et al. (2017) The mark coventry, $M D$, Award: oral antibiotics reduce reinfection after two-stage exchange: A multicenter, randomized controlled trial. Clin Orthop Relat Res 475: 56-61.
33. Diaz Ledezma C, Higuera CA, Parvizi J (2013) Success after treatment of periprosthetic joint infection: A delphi-based international multidisciplinary consensus infection. Clin Orthop Relat Res 471: 2374-2382.

34. AMA (2019) CPT 2020 Professional Edition. Chicago, IL: American Medical Association.

35. Class 2 Device recall stage one hip cement spacer molds.

36. Nodzo SR, Boyle KK, Spiro S, et al. (2017) Success rates, characteristics, and costs of articulating antibiotic spacers for total knee periprosthetic joint infection. Knee 24: 1175-1181.

37. Porter ME (2010) What is value in health care? N Engl J Med 363: 2477-2481. 